\title{
Spatial localization and azimuthal wave numbers of Alfvén waves generated by drift-bounce resonance in the magnetosphere
}

\author{
P. N. Mager and D. Yu. Klimushkin \\ Institute of Solar-Terrestrial Physics (ISTP), Russian Academy of Science, Siberian Branch, Irkutsk, 664033, Russia \\ Received: 27 April 2005 - Revised: 18 October 2005 - Accepted: 21 November 2005 - Published: 23 December 2005
}

\begin{abstract}
Spatial localization and azimuthal wave numbers $m$ of poloidal Alfvén waves generated by energetic particles in the magnetosphere are studied in the paper. There are two factors that cause the wave localization across magnetic shells. First, the instability growth rate is proportional to the distribution function of the energetic particles, hence waves must be predominantly generated on magnetic shells where the particles are located. Second, the frequency of the generated poloidal wave must coincide with the poloidal eigenfrequency, which is a function of the radial coordinate. The combined impact of these two factors also determines the azimuthal wave number of the generated oscillations. The beams with energies about $10 \mathrm{keV}$ and $150 \mathrm{keV}$ are considered. As a result, the waves are shown to be strongly localized across magnetic shells; for the most often observed second longitudinal harmonic of poloidal Alfvén wave $(N=2)$, the localization region is about one Earth radius across the magnetic shells. It is shown that the drift-bounce resonance condition does not select the $m$ value for this harmonic. For $10 \mathrm{keV}$ particles (most often involved in the explanation of poloidal pulsations), the azimuthal wave number was shown to be determined with a rather low accuracy, $-100<m<0$. The $150 \mathrm{keV}$ particles provide a little better but still a poor determination of this value, $-90<m<-70$. For the fundamental harmonic $(N=1)$, the azimuthal wave number is determined with a better accuracy, but both of these numbers are too small (if the waves are generated by $150 \mathrm{keV}$ particles), or the waves are generated on magnetic shells (in $10 \mathrm{keV}$ case) which are too far away. The calculated values of $\gamma / \omega$ are not large enough to overcome the damping on the ionosphere. All these have cast some suspicion on the possibility of the drift-bounce instability to generate poloidal pulsations in the magnetosphere.
\end{abstract}

Keywords. Magnetospheric physics (MHD waves and instabilities) - Space plasma physics (Wave-particle interactions; Waves and instabilities)

Correspondence to: Pavel N. Mager

(p.mager@iszf.irk.ru)

\section{Introduction}

The paper is devoted to studying drift-bounce instability which is suggested as a generation mechanism of azimuthally small-scale, ultra-low frequency waves in the magnetosphere (azimuthal wave numbers $m \gg 1$ ). These waves are represented by poloidally polarized pulsations Pc4 and giant pulsations Pg (Takahashi, 1988, 1996). Drift-bounce instability takes place in the presence of inverted energetic particle populations, i.e. $\partial f / \partial \epsilon>0$, where $f$ is the distribution function and $\epsilon$ is the energy of the particle. Such particle populations associated with high- $m$ pulsations, are often observed in the magnetosphere (Hughes et al., 1978, 1979; Wright et al., 2001; Baddeley et al., 2002). It is assumed that inverted populations emerge as a result of substorm injections (Karpman et al., 1977; Glassmeier et al., 1999).

The features of observed ULF oscillations are probably determined by their sources, in this case by unstable proton populations. As observations show, these oscillations usually have negative azimuthal wave numbers, $m<0$, that is, they propagate in the direction of the positive ion drift. Though they are strongly localized across the magnetic shells in the region about $1 R_{E}$ wide (Takahashi, 1988, 1996), these waves are usually rather broadly distributed in the azimuthal coordinate (Engebretson et al., 1992). In this paper an attempt is taken to find out how the features of unstable proton populations influence these principal features of observed poloidally-polarized pulsations.

The particles can effectively interact with ULF waves, provided that the condition of drift-bounce resonance,

$\omega-m \bar{\omega}_{d}-K \omega_{b}=0$,

is satisfied. Here $K$ is an integer, $\omega_{b}$ is the bounce frequency, and $\bar{\omega}_{d}$ is a bounce averaged drift frequency. Glassmeier et al. (1999) argued that in the case of a mode, highly asymmetric with respect to equator, this resonance condition must be generalized, allowing for non-integer $K$. These asymmetric modes can exist when the conductances of the Northern and Southern Hemispheres are strongly different. This statement 
caused some debate (Mann and Chisham, 2000; Glassmeier, 2000), but we are not going to consider the asymmetric modes and will use the resonance condition in Eq. (1) with integer $K$.

The bounce and drift frequencies both depend on the energy $\epsilon$ of the particle. If the unstable distribution is observed, it is possible to infer the energy of the particles presumably generating the simultaneously observed poloidal high $-m$ wave. Knowing $\epsilon$, it is possible to calculate $\omega_{b}$ and $\bar{\omega}_{d}$ and, after substituting them into Eq. (1), to calculate the azimuthal wave number,

$m=\frac{\omega-K \omega_{b}}{\bar{\omega}_{d}}$.

Then, it is possible to compare this number with the really measured $m$ value. The comparison usually gives good agreement (Glassmeier et al., 1999; Wright et al., 2001; Baddeley et al., 2002). It should be noted that in writing the observed value as $m \pm \Delta m$, the error $\Delta m$ is usually attributed to the measurement rather than to the nature of the waves, tacitly implying that the real $m$ number of the wave has a well-defined value.

It should be mentioned, however, that the resonance condition Eq. (1) is important only as a part of the growth rate expression,

$\gamma \propto\left\langle\frac{\partial f}{\partial \epsilon} \delta\left(\omega-m \bar{\omega}_{d}-K \omega_{b}\right)\right\rangle$

where $\langle\ldots\rangle$ means averaging over the whole velocity space with some weight (see Sect. 2.1). The line of the argumentation described above is valid only in the case when the growth rate $\gamma$ has a very steep and narrow dependence on $m$. In the opposite case, the instability does not select any particular $m$ value, and the whole logic comparising the drift-bounce resonance theory with the experiments fails. To elucidate this issue, it is necessary to perform the integration over the velocity space and to calculate the growth rate as a function of the azimuthal wave number. One of the tasks of this paper is such an integration.

Moreover, according to expression Eq. (2), $\gamma$ is a function of the wave frequency $\omega$. However, for the poloidally polarized wave, the wave frequency coincides with the poloidal eigenfrequency $\Omega_{P}$, which depends on the radial coordinate $L$. Thus, the condition of instability can be satisfied only for a certain $L$ value. Hence, the following questions arise: How strong is the dependence of the growth rate on $m$ and $L$ for the given distribution function? In particular, how sharply are the poloidal Alfvén waves generated by the instability localized across magnetic shells? And what values of the azimuthal wave numbers $m$ can these poloidal Alfvén waves have?

\section{Basic equations}

\subsection{Input equations}

A general expression of the drift-bounce instability growth rate was obtained in a number of papers (e.g. Southwood et al., 1969; Karpman et al., 1977; Chen and Hasegawa, 1988). When the energetic particles are represented by protons, the expression for the growth rate is as follows:

$\gamma=\sum_{K=-\infty}^{\infty} \gamma_{K}$

$\gamma_{K}=\frac{4 \pi}{c^{2}} \frac{q^{2}}{M^{2}} B_{0} L R_{E} \int \frac{\partial f}{\partial \epsilon}\left(1-\frac{\omega_{*}}{\omega}\right) \omega_{b}$

$\times \delta\left(\omega-m \bar{\omega}_{d}-K \omega_{b}\right) G^{2}(\mu, \epsilon) d \mu d \epsilon$,

$G(\mu, \epsilon)=\int_{a}^{b}\left|v_{\|}\right|^{-1} \omega_{d} J_{0} F_{N} \cos I(l) d l$,

$I(l)=\int_{a}^{l}\left|v_{\|}\right|^{-1}\left(\omega-m \omega_{d}\right) d l$.

Here $K$ is an integer, $M, q, \epsilon$ and $\mu=v_{\perp}^{2} / 2 B_{0}$ are the mass, charge, energy and the magnetic moment of a proton, $v_{\perp}, v_{\|}$ are the transverse and the longitudinal components of the particle velocity, $R_{E}$ is the Earth radius, $B_{0}=B_{E} L^{-3}$ is the equatorial magnetic field value, $L$ is the Mcllwain parameter; $B_{E}$ is the magnetic field on the Earth's surface, $\omega$ is the Alfvén wave frequency, $N$ is the longitudinal harmonic number, $\omega_{*}$ is the diamagnetic drift frequency, $\omega_{b}$ is the bounce frequency; $\omega_{d}$ is the drift frequency, $\bar{\omega}_{d}$ is the bounce averaged drift frequency, $f(\epsilon, \mu, L)$ is the distribution function of energetic particles, $J_{0}=J_{0}\left(k_{\perp} v_{\perp} / \omega_{c}\right)$ is a zero order Bessel function, $\omega_{c}$ is the gyrofrequency, $d l$ is the length element along the field line, $a, b$ are points of particle reflection, $m$ is the azimuthal wave number, $k_{\perp}=m / \sqrt{g_{2}}$ is the wave vector transverse component, $g_{i}$ are metric tensor components, the function $F_{N}$ describes the longitudinal structure of the Alfvén wave. Later on we will not take into account finite Larmor radius effects, so $J_{0} \simeq 1$.

Let us introduce a new variable $\xi \equiv \sin \alpha$, where $\alpha$ is the pitch angle. The argument of the delta-function can be written as

$g_{K}(\epsilon, \xi) \equiv \omega-m \bar{\omega}_{d}-K \omega_{b}$.

We denote the solution of the equation

$g_{K}(\epsilon, \xi)=0$

with respect to energy as $\epsilon_{K}$. Then we substitute the integration variables $\epsilon, \mu$ with $\epsilon, \xi$ in Eq. (4), and after that we integrate over $\epsilon$. As a result we have the integral only over the variable $\xi$ :

$$
\begin{aligned}
\gamma_{K} & =\frac{4 \pi}{c^{2}} \frac{q^{2}}{M^{2}} B_{0} L R_{E} \int \frac{\partial f}{\partial \epsilon}\left(1-\frac{\omega_{*}}{\omega}\right) \omega_{b} G^{2}\left(\xi, \epsilon_{K}\right) \\
& \times\left(\frac{\partial g_{K}}{\partial \epsilon}\right)^{-1} \frac{2 \epsilon_{K} \xi}{B_{0}} d \xi
\end{aligned}
$$


where all the derivatives over energy are taken in the point $\epsilon_{K}(\xi)$.

\subsection{Input functions}

For the growth rate calculation we need to know the longitudinal structure of the poloidal Alfvén wave and the distribution function. The longitudinal structure of the Alfvén wave in an axi-symmetric magnetosphere is approximately described in the WKB approximation:

$$
F_{N}=2\left(\sqrt{\frac{g_{2}}{g_{1}}} \frac{A}{\tau_{A}}\right)^{1 / 2} \sin \left(\frac{2 \pi N}{\tau_{A}} \int_{a_{I}}^{l} \frac{d l}{A}\right)
$$

(Leonovich and Mazur, 1993), where $a_{I}, b_{I}$ are magnetoconjugated points of the ionosphere, $A$ is the Alfvén velocity, and $\tau_{A}$ is the transit time with a local Alfvén velocity along the field line "there and back". Although the WKB solution is strictly valid for large $N$ values, qualitatively, it also describes well the wave longitudinal structure for small $N$ numbers considered in this paper. This function is normalized as

$\int_{a_{I}}^{b_{I}} \sqrt{\frac{g_{1}}{g_{2}}} \frac{1}{A^{2}} F_{N}^{2} d l=1$

The Alfvén velocity is modeled by the expression (Leonovich and Mazur, 1993)

$A(L, \theta)=A_{0}(4 / L)^{3 / 2} f_{B}^{1 / 4}(\theta)$,

$f_{B}(\theta)=\left(1+3 \sin ^{2} \theta\right)^{1 / 2} \cos ^{-6} \theta$,

where $\theta$ is the geomagnetic latitude, $A_{0}=10^{3} \mathrm{~km} / \mathrm{s}$.

We used the expression

$f(\epsilon, \mu, L)=f_{0} \exp \left[-\left(\frac{\epsilon-\epsilon_{0}}{\Delta \epsilon}\right)^{2}-\left(\frac{L-L_{0}}{\Delta L}\right)^{2}\right]$

as a distribution function. The most often observed unstable distributions associated with poloidal pulsations have proton energies of about $10 \mathrm{keV}$ (Hughes et al., 1978, 1979; Wright et al., 2001; Baddeley et al., 2002). So, we put $\epsilon_{0}=10 \mathrm{keV}$. For such energies, the inequality $\omega_{*} / \omega \ll 1$ holds in Eqs. (4 and 8 ). The amplitude $f_{0}=10^{3} \mathrm{~km}^{-6} \mathrm{~s}^{3}$ is also chosen in accordance with the observations. Henceforth we will assume $\Delta \epsilon=1 \mathrm{keV} . L_{0}$ and $\Delta L$ can vary considerably as described below.

Bumps with higher energies of about $150 \mathrm{keV}$ are also sometimes observed (e.g. Takahashi et al., 1990). In this case, we take $\epsilon_{0}=150 \mathrm{keV}$. For such values, the amplitude $f_{0}=10 \mathrm{~km}^{-6} \mathrm{~s}^{3}$ and $\Delta \epsilon=10 \mathrm{keV}$. The ratio $\omega_{*} / \omega$ is more significant in this case than in the $\epsilon_{0}=10 \mathrm{keV}$; nonetheless, we will not take this into account.

We omitted the pitch-angle dependency of the distribution function from the calculations, because the observed particle distributions are usually nearly isotropic.

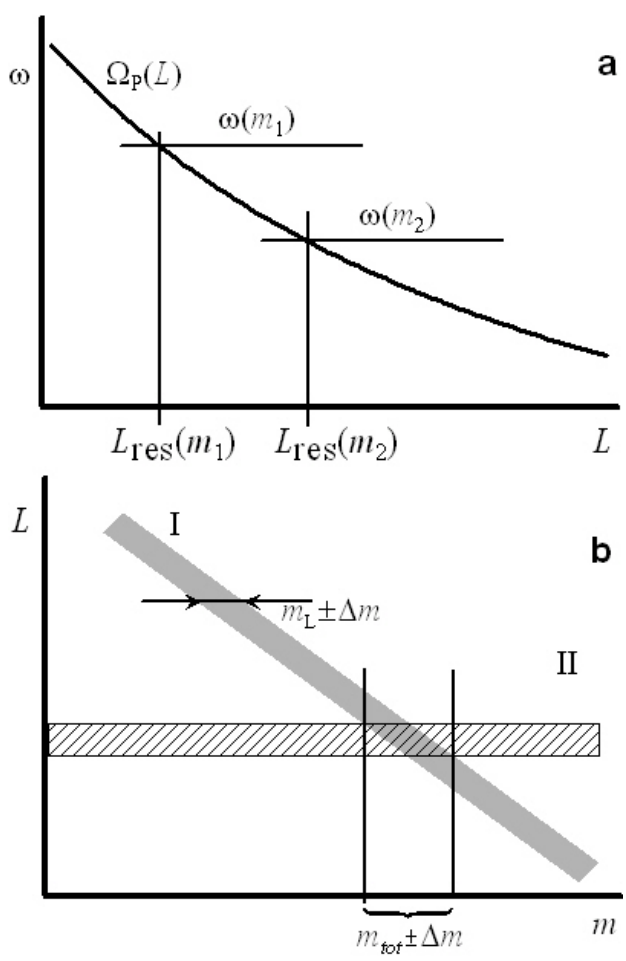

Fig. 1. Sketch of determination of the $L$ and $m$ values of the mode generated by the instability: (a) determination of the mode localization region $L_{0}$ from the drift-bounce resonance condition and dependence of the poloidal eigenfrequency $\Omega_{P}$ on the radial coordinate; (b) selection of $m$ numbers generated by the instability: $m_{L}$ is a value determined on the selected $L$ shell, and $m_{\text {tot }}$ is a total set of $m$-numbers in the whole wave localization region (the intersection of the regions determined by the resonance condition and $\Omega_{P}(L)$ dependence, strip I, and the spatial localization of the energetic particle population, strip II).

\section{Drift-bounce instability growth rate as a function of $L$ and $m$}

\subsection{Formulation of the problem}

Let us clarify the task mentioned at the end of the Introduction. In this paper we study the spatial localization of poloidally-polarized Alfvén waves generated by the driftbounce instability in the magnetosphere. As it is seen from Eq. (4), the instability growth rate for the given distribution function $f(\epsilon, \mu, L)$ and longitudinal wave structure $F_{N}$ depends on the wave frequency $\omega$ and the azimuthal wave number $m$. In a two-dimensionally inhomogeneous magnetosphere, the frequency of the poloidal Alfvén wave $\Omega_{P}$ depends on the radial coordinate $L$ (e.g. Leonovich and Mazur, 1993; Mager and Klimushkin, 2002), hence the growth rate depends on $L$, and the mode located near a shell with a definite coordinate $L_{\text {res }}$, as is shown in Fig. 1a. On the other hand, according to Eq. (1), $\omega$ depends on $m$, so different $m$ will give different locations of the mode $L_{r e s}$. Thus, the driftbounce resonance condition (DBRC) selects the most unstable region as a strip in the $L-m$ plane (Fig. 1b). The width of 


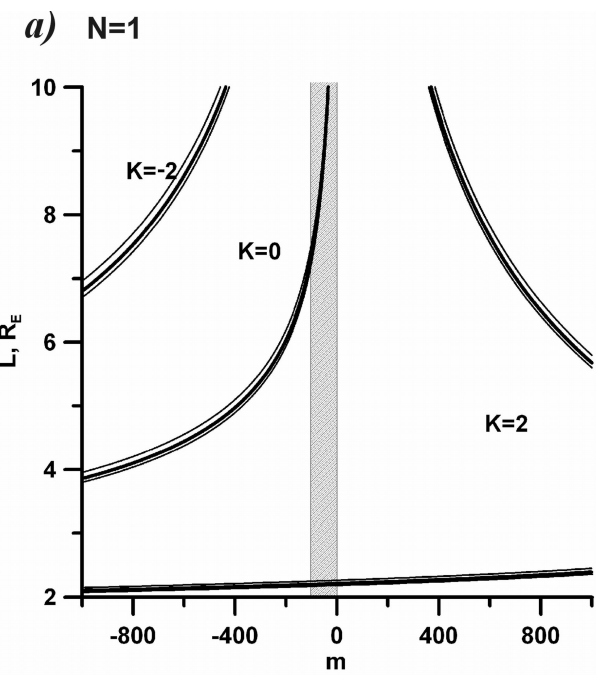

b) $\mathrm{N}=2$

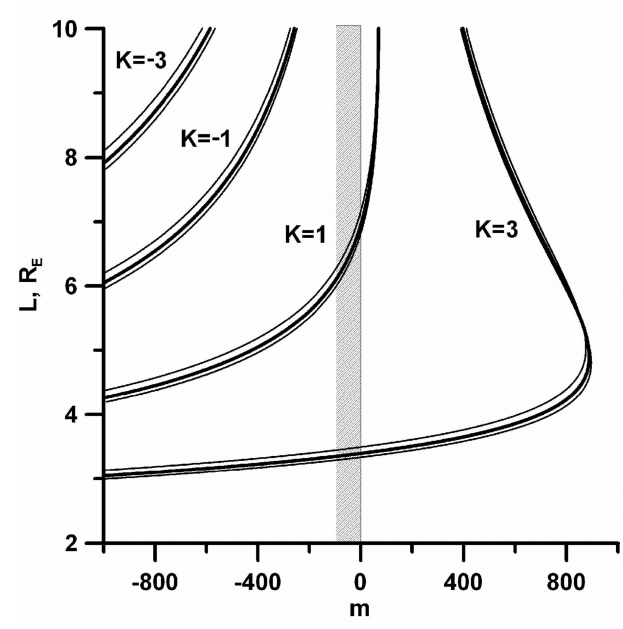

Fig. 2. Location of regions of the most effective instability in $L-m$ plane for various $K$ and $\epsilon \sim 10 \mathrm{keV}$ : (a) $N=1$ case, (b) $N=2$ case. Thick solid lines correspond to the growth rate maximum location, thin lines correspond to an $e$-times smaller growth rate than its maximal value. The shaded region corresponds to the observed values of azimuthal wave numbers $-100<m<0$.

the strip is determined by the width of the distribution function $f$ with respect to particle energy. It also determines the range of $m$ values on a selected $L$ shell $\left(m_{L}\right)$. We are going to elucidate how narrow this strip is, and whether it varies more in the $L$ or $m$ direction.

The dependence of the distribution function $f$ on the radial coordinate $L$ causes an additional $\gamma(L)$ dependence. The location of the particle population forms yet another strip in the $L-m$ plane. The region of the most efficient generation is situated on the intersection of those two strips (see Fig. 1b). Thus, the $f(L)$ dependence can assist the selection of a total set of azimuthal wave numbers $m_{\text {tot }}$ of the most unstable modes in the whole wave localization region (see Fig. 1b).

Different bounce harmonics in the resonance condition Eq. (3) result in different DBRC strips in the $L-m$ plane. If they are well isolated from each other, only some particular $K$ numbers should be taken into account. Quite the reverse, if they are close to each other or intersect, summation over different $K$-numbers must be performed (Pilipenko et al., 1977).

So, after calculation of the instability growth rate for the given distribution function is performed, we can determine the magnetic $L$-shells and the azimuthal wave numbers $m$ on which the poloidal Alfvén waves are most efficiently generated.

\subsection{Contribution of different bounce harmonics}

As is seen from Eqs. (4-6), for odd longitudinal harmonics $N=1,3, .$. , odd bounce components $(K= \pm 1, \pm 3, .$.$) in$ the sum Eq. (3) are equal to zero, while for even harmonics $N=2,4, .$. , even bounce components $(K=0, \pm 2, .$.$) are equal$ to zero (Southwood, 1980). Energy in the integral Eq. (8) is determined by the ratio Eq. (7). The ratio Eq. (7) includes the quantities $\omega_{b}$ and $\bar{\omega}_{d}$, depending on the energy $\epsilon$ and the integration variable $\xi=\sin \alpha$. For odd $N$, the largest contribution to the growth rate is made by particles always located near the equator, i.e. with pitch-angles close to $\pi / 2$. For even $N$, in turn, the largest contribution is made by the particles traveling the largest distances between the turning points, which corresponds to the smallest pitch-angles $\alpha_{\text {min }}$ (Southwood, 1980):

$\sin \alpha_{\min }=f_{B}^{1 / 2}\left(\theta_{I}\right)$,

$\theta_{I}=\arccos \left(\frac{L_{I}}{L}\right)^{1 / 2}$

where $L_{I}$ is the radius of the ionosphere's external border in units of the Earth's radius, $R_{E} ; \theta_{I}$ is the geomagnetic latitude of the point where the magnetic field line crosses the top of the ionosphere. If we do not take into account the spatial localization of distribution function $(f(L)=$ const $)$, the growth rate is maximal with energy values $\epsilon_{\text {eff }}$ corresponding to the function maximum $\partial f / \partial \epsilon$. For our model distribution function Eq. (10), $\epsilon_{e f f}=\epsilon_{0}-\Delta \epsilon / \sqrt{2}$. Thus, substituting into Eq. (7) the energy quantity $\epsilon_{\text {eff }}$ and the pitchangle $\alpha=\pi / 2$ for $N=1$ or $\alpha_{\min }$ for $N=2$, we can determine at which $L$-shells and with which $m$ values the growth rate is the largest. The borders of region of the most effective instability are approximately determined from Eq. (7) for particle energies corresponding to the function $\partial f / \partial \epsilon$ values $e$-times smaller than its maximal value.

The most frequently observed poloidal oscillations are usually the first or the second longitudinal harmonics, $N=1,2$. The DBRC regions are shown in Figs. 2a and 3a for $N=1$ and in Figs. $2 \mathrm{~b}$ and $3 \mathrm{~b}$ for $N=2$, as regions between thin solid lines. It is seen in Figs. 2 and 3 that the instability regions for different $K$ values are mutually isolated. It means that in considering some region on the $L-m$ diagram, only some selected $K$-values are essential.

Observed poloidal waves usually propagate westward $(m<0)$, and have $|m|$-values from 50 to 100 . For such 


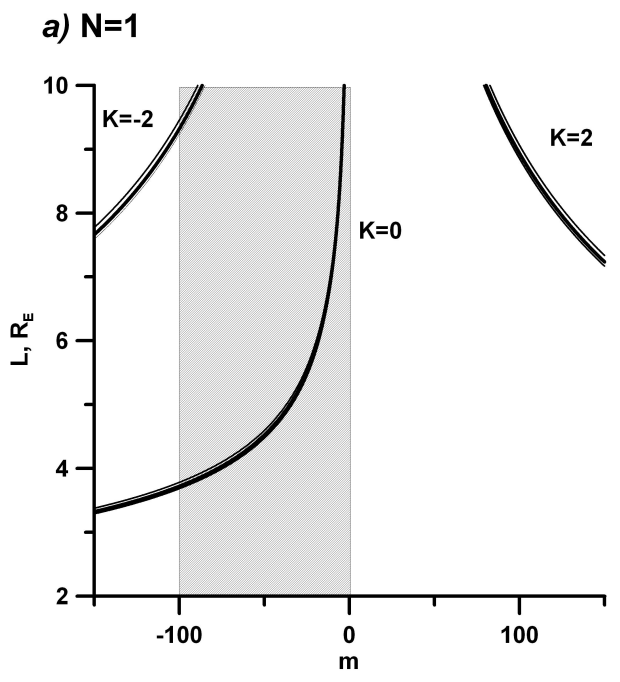

b) $\mathrm{N}=2$

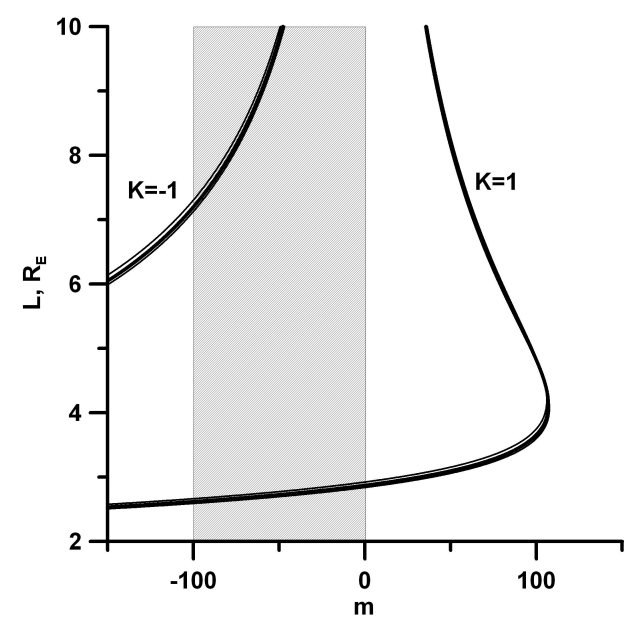

Fig. 3. Location of regions of the most effective instability in $L-m$ plane for various $K$ and $\epsilon \sim 150 \mathrm{keV}$ : (a) $N=1$ case, (b) $N=2$ case. Thick solid lines correspond to the growth rate maximum location, thin lines correspond to an $e$-times smaller growth rate than its maximal value. The shaded region corresponds to the observed values of azimuthal wave numbers $-100<m<0$.

$m$ values and energies $\epsilon \sim 10 \mathrm{keV}$, only components with $K=0,2$ contribute to the growth rate for the fundamental standing harmonic $(N=1)$, and components with $K=1,3$ do the same for the second harmonic $(N=2)$, because the components with $K<0$ for $N=1$ and with $K<1$ for $N=2$ are essential only for $m<-100$, and the components with $K>2$ for $N=1$ and with $K>3$ for $N=2$ are essential only for $m>0$. Moreover, the energetic particles with unstable distributions with $(\partial f / \partial \epsilon>0)$ are observed in magnetospheric regions with $L \sim 6-10 R_{E}$. It follows that for $N=1$ only the component $K=0$ (drift resonance) can contribute to the growth rate, and for $N=2$ the contribution can be made by the component $K=1$ (drift-bounce resonance). For the same reason, in the $\epsilon \sim 150 \mathrm{keV}$ case, for $N=1$, the contribution to the growth rate can be made only by the component with

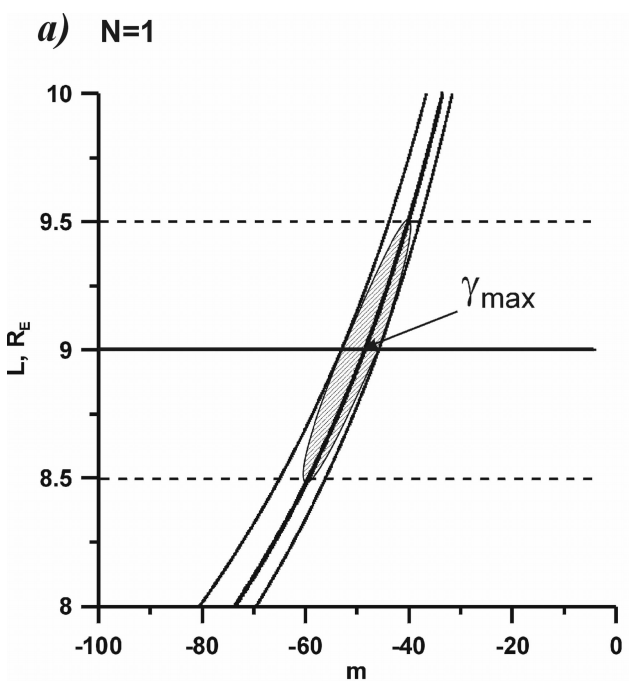

b) $\mathrm{N}=2$

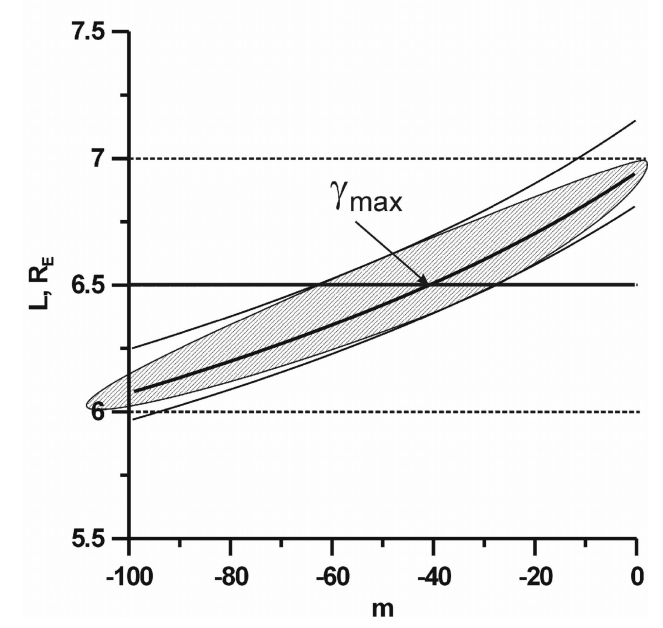

Fig. 4. Location of the regions of the most effective instability in $L-m$ plane for $\epsilon \sim 10 \mathrm{keV}$ : (a) for $K=0$ and $N=1$,(b) for $K=1$ and $N=1$. Thick solid lines correspond to the growth rate maximum location, thin ones correspond to an $e$-times smaller growth rate than its maximal value. Shaded areas show regions of the most effective instability for the spatially inhomogeneous distribution. The solid horizontal line indicates the maximum of distribution function, the dotted lines indicates an $e$-times smaller value.

$K=0$ (drift resonance), and for $N=2$, by the component $K=-1$ (drift-bounce resonance).

\section{3 $\gamma=\gamma(L, m)$ : qualitative considerations and numerical calculations}

The $\epsilon \sim 10 \mathrm{keV}$ case. Figure 4 shows the important instability regions from Fig. 2 in more detail. For both $N=1,2$ longitudinal harmonics, the DBRC region (region between thin solid lines in Fig. 4) appeared to be strongly localized across $L$ shells: for a certain value of $m$ their width is less than $0.5 R_{E}$. As this takes place, for $N=1$ the DBRC region in the $L-m$ plane is strongly localized with respect to the azimuthal wave numbers: $m_{L}$ (the $m$-value at a certain $L$-shell) 
is determined with a \pm 5 accuracy. On the contrary, for the often observed second harmonic $(N=2)$ the localization with respect to $m$ is weak: $m_{L}$ is defined with an uncertainty larger than 30; the DBRC region runs almost parallel to the $m$ axis in the $L-m$ plane. The weak $m$-dependence of the growth rate in the $N=2$ case is explained by the smallness of the term $\omega_{d}$ in resonance condition Eq. (7), for the observed values of the wave period of $\sim 100$ seconds, $m$-number from -50 to -100 , and the proton energies of about $10 \mathrm{keV}$ for $K=1$.

Let us now take into account the spatial inhomogeneity of the particle distribution, that is, the $f(L)$ dependence. In the most interesting case the population is localized near those $L-$ shells where the DBRC regions are situated (see Figs. 4a, b). These regions are situated near $L=9$ in the $N=1$ case and near $L=6.5 R_{E}$ in the $N=2$ case. Let the width of the localization region be $1 R_{E}$. So, in our model distribution function Eq. (10), we will assume $L_{0}=9$ for $N=1$ and $L_{0}=6.5 R_{E}$ for $N=2, \Delta L=0.5 R_{E}$. As a result, the region of the most effective generation of poloidal Alfvén waves, given the spatial inhomogeneity of the population (the shaded region in Fig. 4), is located between the magnetic shells $L=8-9$ for $N=1$, and between $L=6-7 R_{E}$ for $N=2$, thus, $m_{\text {tot }}$ (azimuthal wave numbers $m_{\text {tot }}$ in the entire wave localization region) is enclosed in the interval from -100 to 0 . It should be mentioned that poloidal waves can exist only with the values $|m| \gg 1$ (Leonovich and Mazur, 1997; Klimushkin et al., 2004), and that also limits the value of $m$ and the instability region width on $L$. The maximum growth rate $\gamma_{\max }$ is reached when $L=9$ and $m_{L}=-50 \pm 5$ for $N=1$, and for $N=2$ when $L=6.5 R_{E}$ and $m_{L}=-40 \pm 15$.

The results of the numerical calculation of the instability growth rate $\gamma$, as a function of $L$ and $m$, are presented in Fig. 5. The maximum growth rate for $10 \mathrm{keV}$ is $\gamma / \omega=2.4 \cdot 10^{-1}$ for $N=1$, and $\gamma / \omega=2.2 \cdot 10^{-3}$ for $N=2$. Note a slight difference between locations of the instability regions shown in Fig. 5 (numerically calculated) and in Fig. 4 (analytically determined). It is mainly an apparent difference, because it is the ratio $\gamma / \omega$ that is shown in Fig. 5, where the eigenfrequency $\omega$ itself strongly depends on $L$.

The $\epsilon \sim 150 \mathrm{keV}$ case. The DBCR region is strongly localized across $L$ shells, as well as $m$, for both $N$ harmonics. The $m_{L}$ value is determined with an accuracy of \pm 2 for $N=1$ and of \pm 3 for $N=2$. It must be noted, however, that such a high accuracy is determined by the small $\Delta \epsilon$ value chosen in this paper. Inserting in Eq. (10) the width $\Delta L=0.5 R_{E}$ and the maximum location $L_{0}=6.5$ for $N=1$ and $L_{0}=8 R_{E}$ for $N=2$, we find the location of the most unstable region (the shaded region in Fig. 6) for $N=1$ between the magnetic shells $L=6$ and $7 R_{E}$, and for $N=2$ between $L=7.5$ and $8.5 R_{E}$. Thus, $m_{\text {tot }}$ is confined to the interval -20 to -10 for $N=1$ and -90 to -70 for $N=2$. The maximum growth rate $\gamma_{\max }$ is reached when $L=6.5$ and $m=-14 \pm 2$ for $N=1$, and for $N=2$ when $L=8 R_{E}$ and $m=-78 \pm 3$.

The results of numerical calculation of $\gamma(L, m)$ for energies $150 \mathrm{keV}$ are presented in Fig. 7. The largest value of the
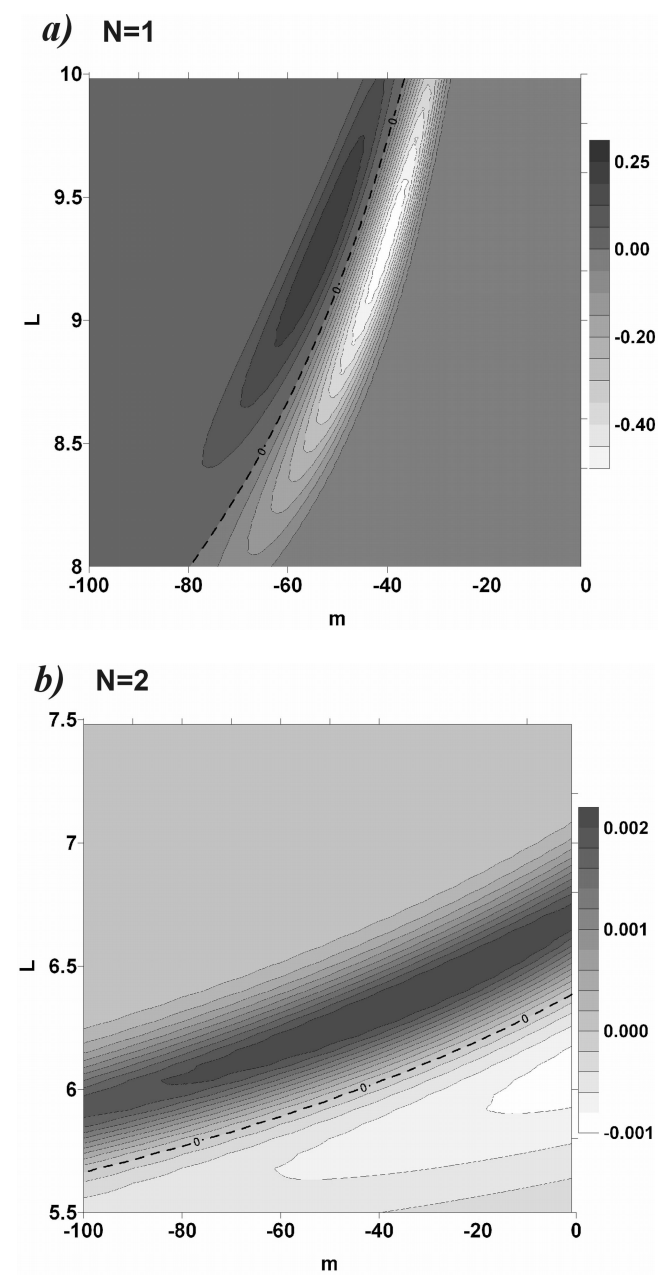

Fig. 5. Numerically calculated growth rate dependency $\gamma / \omega$ on radial coordinate $L$ and azimuthal wave number $m$ for $\epsilon_{0}=10 \mathrm{keV}$ : (a) in $N=1$ case, (b) in $N=2$ case. Dotted line corresponds to the zero growth rate, $\gamma=0$.

growth rate is $\gamma / \omega=3 \cdot 10^{-2}$ for $N=1$, and $\gamma / \omega=2 \cdot 10^{-3}$ for $N=2$.

\section{Discussion}

The width of the distribution function $f$ with respect to particle energy is the crucial factor of the drift-bounce instability growth rate, because the values of drift $\omega_{d}$ and bounce $\omega_{b}$ frequencies depend on the energy $\epsilon$. The variation of these frequencies appears to be quite narrow because of the small width of the function $f(\epsilon)$. Consequently, the wave frequency range (with fixed $m$ and $K$ ) which satisfies the resonance condition Eq. (7) is also quite narrow. On the other hand, the eigenfrequency of standing Alfvén waves depends on the radial coordinate $L$. Thus, the condition of the driftbounce resonance can be satisfied in the vicinity of a certain $L$-shell. Moreover, there is another factor, namely, the beam localization in the magnetosphere. The highest growth rate takes place when these two factors select the same $L$ 


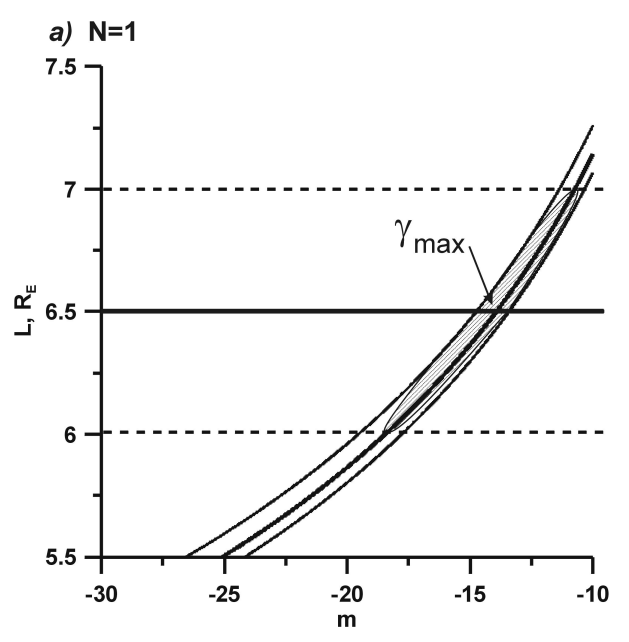

b) $\mathrm{N}=2$

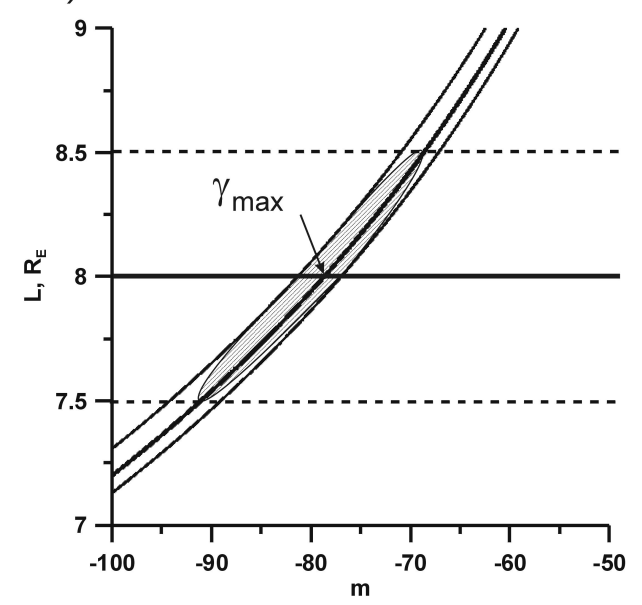

Fig. 6. Location of the regions of the most effective instability in $L-m$ plane for $\epsilon \sim 150 \mathrm{keV}$ : (a) for $K=-2$ and $N=1$, (b) for $K=-1$ and $N=2$. Thick solid lines correspond to the growth rate maximum location, thin lines correspond to an $e$-times smaller growth rate than its maximal value. Shaded areas show regions of the most effective instability for the spatially inhomogeneous distribution. The solid horizontal line indicates the maximum of distribution function, the dotted lines indicates an $e$-times smaller value.

shells. For the particles with energies $\epsilon \sim 10 \mathrm{keV}$ and waves with $|m| \sim 50-100$, the beam should be located near the magnetic shell with $L=6.5$ for the second longitudinal harmonic $(N=2)$ and $L=9$ for the fundamental one $(N=1)$. For particles with energies $\epsilon \sim 150 \mathrm{keV}$ it is $L=8$ for $N=2$ and $L=6.5$ for $N=1$.

As it is seen from our analytical and numerical calculations, the localization width is less than $1 R_{E}$. This can explain the narrow localization of the observed poloidal pulsation. For example, Engebretson et al. (1992) found that poloidal wave events observed in situ are strongly localized across the magnetic shells (scale of $1 R_{E}$ ). On the other hand, other explanations exist. Poloidally polarized waves can be enclosed in the resonator in magnetospheric regions where the function $\Omega_{P}(L)$ has a local extremum (e.g. Vetoulis and

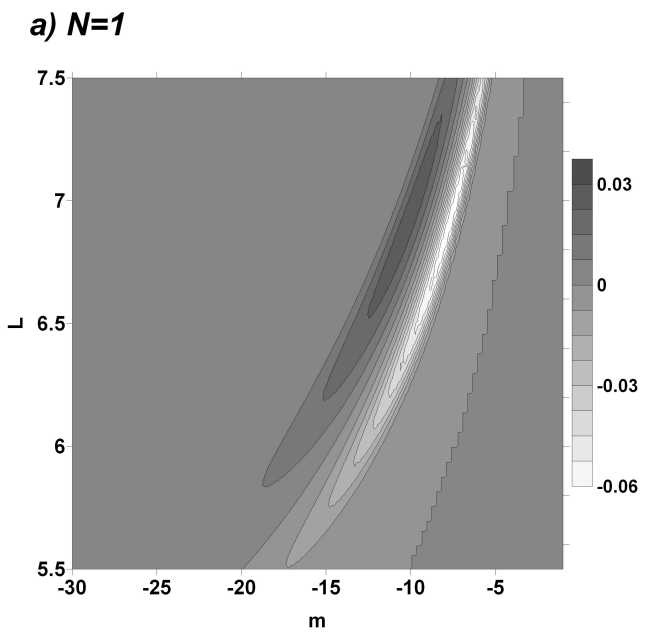

b) $N=2$

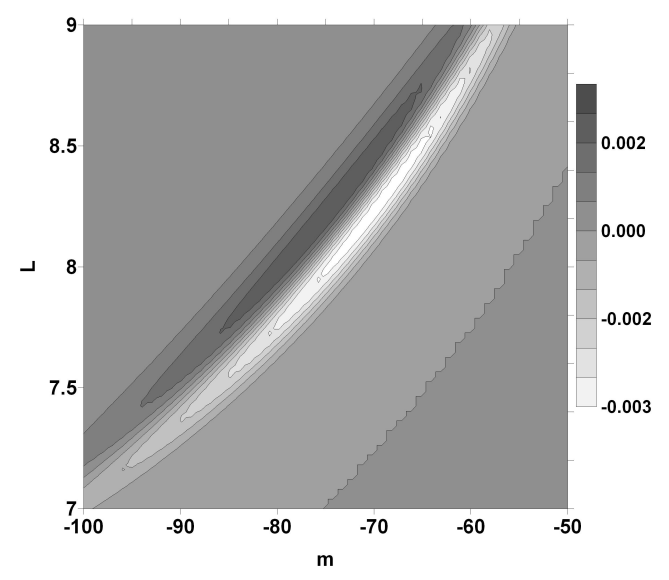

Fig. 7. Numerically calculated growth rate dependency $\gamma / \omega$ on radial coordinate $L$ and azimuthal wave number $m$ for $\epsilon_{0}=150 \mathrm{keV}$ : (a) in $N=1$ case, (a) in $N=2$ case.

Chen, 1996; Klimushkin et al., 2004), which has some experimental support (Denton et al., 2003). The resonator width is also $1 R_{E}$. Outside such extreme regions, a high- $m$ wave propagates across $L$-shells, changing its polarization from poloidal to toroidal (Leonovich and Mazur, 1993); but if the damping rate on the ionosphere is much larger than the instability growth rate, then the wave is attenuated, remaining poloidally polarized (Klimushkin, 2000), and also will be observed as a poloidal wave localized in a narrow range of $L$ values.

The next important issue is the $\gamma(m)$ dependence. As it was mentioned in the Introduction, a comparison of the instability theory with the observations tacitly implies that $m$ is a well-defined value, and the $\gamma(m)$ dependence is very sharp and narrow, like a delta-function. Our calculations show, however, that the drift-bounce instability practically does not single out a particular value of $m$. It is especially evident for the oscillations with $N=2$, generated by $10 \mathrm{keV}$ particles (the most often observed harmonic and the most often suggested energy): $m_{L}$ can be determined with an accuracy 
of \pm 15 , and $m_{\text {tot }}>-100$ can be generated. We used quite a narrow distribution function in our calculations: $\Delta \epsilon \sim 2 \mathrm{keV}$; for wider distributions, the azimuthal wave number will be determined with an even smaller accuracy. Thus, the usual case for the drift-bounce instability as a generation mechanism of poloidally-polarized pulsations Pc4 and Pg (which are often identified with the second longitudinal harmonic, $N=2$ ) loses its meaning.

There are additional limitations on the $m$ number that follow from the fact that the condition $|m| \gg 1$ is necessary, but not sufficient for the poloidal polarization of the wave (Klimushkin et al., 2004). Even the values as high as $|m| \simeq 40$ can be not high enough for the poloidal polarization. In order to increase the $|m|$ number of the mode generated by the instability, the beam of energetic particles must move closer to Earth. But in this case additional constraints arise, because particles usually cannot penetrate into the plasmasphere. Due to a large number of these limitations, some doubts are cast upon the possibility of those $10 \mathrm{keV}$ populations to generate poloidal pulsations. Anyway, the corridor of possibilities is rather narrow.

For the fundamental harmonic of poloidal oscillations $(N=1)$, the azimuthal wave number $m$ can be determined with greater accuracy: for the distribution function with $\epsilon=10 \mathrm{keV}, m_{L}$ is determined with the accuracy of \pm 5 and $m_{\text {tot }}$ with the accuracy of \pm 10 , but the region of their localization with observed values $|m| \sim 50-100$ is located in the distant part of the magnetosphere with $L>9 R_{E}$.

If pulsations are excited by higher energy distributions, like protons with $\epsilon \sim 150 \mathrm{keV}$ considered here, the situation is almost the same. The second harmonic can have $m_{\text {tot }}$ values from -90 to -70 , though $m_{L}$ is determined with the accuracy \pm 3 . These oscillations can have reasonable $m$ values only if particles are localized near $L \simeq 8$. In the vicinity of the geosynchronous orbit, such particles will generate waves with $|m|>100$. It should be noted that the results are sensitive to the dependence of the Alfvén velocity on the radial coordinate. A weaker $A(L)$ dependence than discussed in our paper will result in increasing the $|m|$ number of the generated wave on the same magnetic shell, and a stronger one will do the opposite.

In the $N=1$ case, the instability region is situated in the vicinity of the geosynchronous orbit (Figs. 6a and 7a), but only rather low- $m$ oscillations $\left(m_{\text {tot }} \sim-15 \pm 5\right)$ will be generated there. Such $m$ values are not large enough for waves to be poloidally polarized (Klimushkin et al., 2004). Though such factors as finite plasma pressure, transverse and longitudinal equilibrium current can provide more possibilities for moderately-high- $m$ poloidal waves to exist (Klimushkin et al., 2004; Klimushkin and Mager, 2004a).

Thus, drift-bounce instability hardly explains the observed values of $|m|$. Moreover, there are problems with the explanation of the azimuthal wave number sign. For example, as is seen from Fig. $2 b, 10 \mathrm{keV}$ particles can generate the second standing harmonic with $m>0$ : near $L \simeq 7.5 R_{E}$ for $K=1$. This also is true for $150 \mathrm{keV}$ particles (see Fig. 3b). Thus, the drift-bounce instability cannot explain the fact that observed poloidal Alfvén waves usually (though not always) have negative $m$ values, propagating in the direction of the positive ions drift.

The values of the growth rate are $\gamma / \omega \sim 10^{-1}-10^{-2}$ for $N=1$ and $\gamma / \omega \sim 10^{-3}$ for $N=2$. These values are calculated using realistic densities of the energetic particles, in agreement with the observed values. We used rather sharp functions $f(\epsilon)$, broader (perhaps, more realistic) distributions will give even smaller growth rates. The instability can be an effective generation mechanism if the growth rate is substantially larger than the damping rate on the ionosphere, which is typically 10 or 100 times smaller than the wave frequency. Hence, some doubts are cast upon the capability of the instability to overcome the attenuation on the ionosphere.

\section{Conclusions}

First, let us describe the methodological approach of this work.

(i) The way in which drift-bounce instability defines localization of poloidal Alfvén oscillations across magnetic shells is found. The wave frequency is determined from the resonance condition $\omega-m \bar{\omega}_{d}-K \omega_{b}=0$. As this takes place, the frequency of the generated wave $\omega$ must coincide with the poloidal eigenfrequency, which is a function of the radial coordinate. Further, $\omega_{d}$ and $\omega_{b}$ depend on the particle energy, hence the range of $L$ shells of generated waves is determined by the width of the distribution function with respect to energy.

(ii) The distribution function also determines azimuthal wave numbers of generated oscillations. First, since the azimuthal wave number is involved in the resonance condition, the radial coordinate of the generated pulsations is a function of $m$. So, if $L$ is fixed, the range of the azimuthal wave numbers is ultimately determined by the width of the function $f(\epsilon)$. Then, waves must be generated on those magnetic $L$ shells where the particles are located. By this means, azimuthal wave numbers of the pulsation in the whole localization region are determined.

Let us describe the main results of our work.

1. It has been found that the function $f(\epsilon)$ itself determines the location and width of the generation region of poloidal Alfvén waves. If particles with energies $\sim 10 \mathrm{keV}$ are responsible for the excitation, the waves with reasonable $m$ values have $L \simeq 8 R_{E}$ (the fundamental longitudinal harmonic, $N=1$ ) or $L \simeq 6.5 R_{E}$ (the second harmonic, $N=2$ - the most often observed case). For energies $\sim 150 \mathrm{keV}$, the waves are situated near $L \simeq 6.5 R_{E}$ ( $N=1$ case) or $L \simeq 8 R_{E}(N=2$ case $)$. The width of the localization region is about 1 Earth radius in each case. This is in agreement with an observed narrow localization of the poloidal pulsations (Takahashi, 1988, 1996; Engebretson et al., 1992). However, there are other explanations of this phenomenon. 
2. The predicted azimuthal wave numbers are presented in the following:

\begin{tabular}{|c|c|c|}
\hline 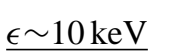 & $N=1$ & $N=2$ \\
\hline$\overline{m_{\text {tot }}}$ & $-50 \pm 10$ & $-50 \pm 50$ \\
\hline$m_{L}$ & $-50 \pm 5$ & $-40 \pm 15$ \\
\hline$\underline{\epsilon \sim 150 \mathrm{keV}}$ & $N=1$ & $N=2$ \\
\hline$m_{\text {tot }}$ & $-15 \pm 5$ & $-80 \pm 10$ \\
\hline$m_{L}$ & $-14 \pm 2$ & $-78 \pm 3$ \\
\hline
\end{tabular}

Here $m_{\text {tot }}$ and $m_{L}$ are the azimuthal wave numbers in the entire wave localization region and on the $L$ shell where the growth rate reaches its maximum value, respectively.

As we see, for the most often observed second longitudinal harmonic, the drift-bounce instability does not select any definite value of the azimuthal wave number $m$. The situation is a little better for $150 \mathrm{keV}$ particles than for the $10 \mathrm{keV}$ particles, especially if the waves on a fixed $L$ shell are considered, but it is not clear as to whether it has more sense than $m$ in the entire wave localization region. Anyway, the usual argumentation employed in the comparison of the theory with the experiments is invalid, since it tacitly assumes that $m$ is well defined by the drift-bounce resonance condition. Observations often give higher accuracy, \pm 5 (e.g. Glassmeier et al., 1999; Wright et al., 2001; Baddeley et al., 2002). This raises the questions: What determines the azimuthal wave number in the most characteristic case $(N=2)$ ? Is the error in the $m$ determination due to measurement or due to the wave nature? Is $m$ experimentally determined on some selected $L$ shell or in the entire wave localization region?

Moreover, the instability in both the $10 \mathrm{keV}$ and $150 \mathrm{keV}$ cases can generate waves of both signs of $m$, whereas the majority of observed poloidal waves have negative signs.

3 . Growth rates are calculated for different particle energies and longitudinal wave numbers using realistic particle densities. The results are $\gamma / \omega \sim 10^{-1}-10^{-2}$ for $N=1$ and $\gamma / \omega \sim 10^{-3}$ for $N=2$. It is the same order of magnitude as the damping rate on the ionosphere. Hence, it is not clear whether the instability can overcome the attenuation.

It should also be added that poloidal modes in the magnetosphere quickly transform into toroidal modes (Leonovich and Mazur, 1993, 1997; Klimushkin et al., 2004). If the instability condition is satisfied for the time of the transformation, then the toroidal rather than the poloidal amplitude increases (Klimushkin, 2000; Klimushkin and Mager, 2004b).

Thus, drift-bounce instability as a generation mechanism produces more questions than answers. It means that other generation mechanisms can be suggested. In particular, an azimuthally drifting cloud of substorm-injected particles can be a direct driver of the wave, as it represents a current that serves as a right-hand side of the wave equation, as was first proposed by Zolotukhina (1974) and Gul'elmi and Zolotukhina (1980).
Acknowledgements. The work was supported by RFBR grants 03-05-64545a and 04-05-64321a.

Topical Editor T. Pulkkinen thanks S. Buchert and W. J. Hughes for their help in evaluating this paper.

\section{References}

Baddeley, L. J., Yeoman, T. K., Wright, D. M., Davies, J. A., Trattner, K. J., and Roeder, J. L.: Morning sector drift-bounce resonance driven ULF waves observed in artificially-induced HF radar backscatter, Ann. Geophys., 20, 1487-1498, 2002,

\section{SRef-ID: 1432-0576/ag/2002-20-1487.}

Chen, L. and Hasegawa, A.: On magnetospheric hydromagnetic waves exited by energetic ring-current particles, J. Geophys. Res., 93, 8763-8767, 1988.

Denton, R. E., Lessard, M. R., and Kistler, L. M.: Radial localization of magnetospheric guided poloidal Pc 4-5 waves, J. Geophys. Res., 108(A3), 1105, doi:10.1029/2002JA009679, 2003.

Engebretson, M. J., Murr, D. L., Erickson, K. N., Strangeway, R. J., Klumpar, D. M., Fuselier, S. A., Zanetti, L. J., and Potemra, T. A.: The spatial extent of radial magnetic pulsations events observed in the dayside near synchronous orbit, J. Geophys. Res., 97, 13741-13 758, 1992.

Glassmeier, K.-H.: Reply to the comment by I. R. Mann and G. Chisham, Ann. Geophys., 18, 167-169, 2000,

SRef-ID: 1432-0576/ag/2000-18-167.

Glassmeier, K.-H., Buchert, S., Motschmann, U., Korth, A., and Pedersen, A.: Concerning the generation of geomagnetic giant pulsations by drift-bounce resonance ring current instabilities, Ann. Geophys., 17, 338-350, 1999,

SRef-ID: 1432-0576/ag/1999-17-338.

Gul'elmi, A. V. and Zolotukhina, N. A.: Excitation of Alfvén oscillations of the magnetosphere by the asymmetric ring current, Issled. geomagn. aeron. i fiz. Solntsa (in Russian), 50, 129-137, 1980.

Hughes, W. J., Southwood, D. J., Mauk, B., McPherron, R. L., and Barfield, J. N.: Alfvén waves generated by an inverted plasma energy distribution, Nature, 275, 43-44, 1978.

Hughes, W. J., McPherron, R. L., Barfield, J. N., and Mauk, B.: A compressional Pc 4 pulsation observed by three satellites in geostationary orbit near local midnight, Planet. Space Sci., 27, 821-840, 1979.

Karpman, V. I., Meerson, B. I., Mikhailovsky, A. B., and Pokhotelov, O. A.: The effects of bounce resonances on wave growth rates in the magnetosphere, Planet. Space. Sci., 25, 573585, 1977.

Klimushkin, D. Yu.: The propagation of high- $m$ Alfvén waves in the Earths magnetosphere and their interaction with high-energy particles, J. Geophys. Res., 105, 23 303-23 310, 2000.

Klimushkin, D. Yu. and Mager, P. N.: The structure of lowfrequency standing Alfvén waves in the box model of the magnetosphere with magnetic field shear, J. Plasma Phys., 70, 379-395, 2004a.

Klimushkin, D. Yu. and Mager, P. N.: The spatio-temporal structure of impulse-generated azimuthal small-scale Alfven waves interacting with high-energy charged particles in the magnetosphere, Ann. Geophys., 22, 1053-1060, 2004b,

SRef-ID: 1432-0576/ag/2004-22-1053.

Klimushkin, D. Yu., Mager, P. N., and Glassmeier, K.-H.: Toroidal and poloidal Alfvén waves with arbitrary azimuthal wave numbers in a finite pressure plasma in the Earths magnetosphere, 
Ann. Geophys., 22, 267-288, 2004,

SRef-ID: 1432-0576/ag/2004-22-267.

Leonovich, A. S. and Mazur, V. A.: A theory of transverse smallscale standing Alfvén waves in an axially symmetric magnetosphere, Planet. Space Sci., 41, 697-717, 1993.

Leonovich, A. S. and Mazur, V. A.: A model equation for monochromatic standing Alfvén waves in the axially-symmetric magnetosphere, J. Geophys. Res., 102, 11 443-11 456, 1997.

Mager, P. N. and Klimushkin, D. Yu.: Theory of azimuthally smallscale Alfvén waves in an axisymmetric magnetosphere with small but finite plasma pressure, J. Geophys. Res., 107(A11), 1356, doi:1029/2001JA009137, 2002.

Mann, I. R. and Chisham, G.: Comment on: Concerning the generation of geomagnetic giant pulsations by drift-bounce resonance ring current instabilities, edited by: Glassmeier, K.-H. et al., Ann. Geophys., 17, 338-350, 1999, Ann. Geophys., 18, 161166, 2000,

SRef-ID: 1432-0576/ag/2000-18-161.

Pilipenko, V. A., Pokhotelov, O. A., and Feigin, F. Z.: Effect of bounce resonance on Alfvén-wave excitation outside the plasmasphere, Geomagnetizm i Aeronomiia (in Russian), 17, 894-899, 1977.

Southwood, D. J., Dungey, J. W., and Etherington, R. J.: Bounce resonant interaction between pulsation and trapped particles, Planet. Space Sci., 17, 3549-3561, 1969.
Southwood, D. J.: Low frequency pulsation generation by energetic particles, J. Geomagn. Geoelectr., Suppl. II, 32, 75-88, 1980.

Takahashi, K.: Multisatellite studies of ULF waves, Adv. Space Res., 8(9-10), (9)427-(9)436, 1988.

Takahashi, K., McEntire, R. W., Lui, A. T. Y., and Potemra, T. A.: Ion flux oscillation associated whith a radially polarized transverse Pc5 magnetic pulsations, J. Geophys. Res., 95, 3717-3731, 1990.

Takahashi, K.: New observations, new theoretical results and controversies regarding Pc3-5 waves, Adv. Space Res., 17, 10631071, 1996.

Vetoulis, G. and Chen, L.: Kinetic theory of geomagnetic pulsations, 3, Global analysis of drift Alfvén-ballooning modes, J. Geophys. Res., 101, 15 441-15 456, 1996.

Wright, D. M., Yeoman, T. K., Rae, I. J., Storey, J., Stockton-Chalk, A. B., Roeder, J. L., and Trattner, K. J.: Ground-based and Polar spacecraft observations of a giant $(\mathrm{Pg})$ pulsation and its associated source mechanism, J. Geophys. Res., 106, 10 837-10 852, 2001.

Zolotukhina, N. A.: On excitation of Alfvén waves in the magnetosphere by a moving source, Issled. geomagn. aeron. i fiz. Solntsa (in Russian), 34, 20-23, 1974. 